\title{
HOW IS NON-KNOWLEDGE REPRESENTED IN ECONOMIC THEORY?
}

\author{
EKATERINA SVETLOVA* and HENK VAN ElsT ${ }^{\dagger}$ \\ ${ }^{1}$ Fakultät I: Betriebswirtschaft und Management, Karlshochschule International University \\ Karlstraße 36-38, 76133 Karlsruhe, Germany
}

September 10, 2012

\begin{abstract}
In this article, we address the question of how non-knowledge about future events that influence economic agents' decisions in choice settings has been formally represented in economic theory up to date. To position our discussion within the ongoing debate on uncertainty, we provide a brief review of historical developments in economic theory and decision theory on the description of economic agents' choice behaviour under conditions of uncertainty, understood as either (i) ambiguity, or (ii) unawareness. Accordingly, we identify and discuss two approaches to the formalisation of non-knowledge: one based on decision-making in the context of a state space representing the exogenous world, as in Savage's axiomatisation and some successor concepts (ambiguity as situations with unknown probabilities), and one based on decision-making over a set of menus of potential future opportunities, providing the possibility of derivation of agents' subjective state spaces (unawareness as situation with imperfect subjective knowledge of all future events possible). We also discuss impeding challenges of the formalisation of non-knowledge.
\end{abstract}

\section{Introduction}

The recent economic crisis once again drew attention to the insufficient ability of modern economic theory to properly account for uncertainty and imperfect knowledge: neglect of these issues is argued to be one of the reasons for the failure of the economic profession in the difficult times of 2007-2009; cf. The Economist (2007) [20], Colander et al (2009) [10], Taleb (2010) [72], Akerlof and Shiller (2009) [2], and Svetlova and Fiedler (2011) [70]. Next to the voices from the inside of the profession, there is the related criticism from neighbouring disciplines such as, e.g., economic sociology; cf. Beckert (1996) [3], and Esposito (2007, 2010) [25, 26]. The impression arises that economists are utterly ignorant: they supposedly do not pay (enough) attention to the issues which the rest of the world consider to be most crucial for economic life. We asked ourselves if this ignorance is indeed a part of scientific practice in economics. Is it correct that nobody has properly tackled the issue of true uncertainty and imperfect knowledge since Knight (1921) [45] and Keynes (1921) [42] during the post-WW I twentieth century?

In this article, we aim to arrive at a more differentiated judgement. Based on a review of the literature, we classify the developments in economics and decision theory that refer to uncertainty and imperfect knowledge. We identify three major directions that deal with these issues in economics, specifically risk, uncertainty as ambiguity, and uncertainty as unawareness. However, it should be stressed that our goal is not a detailed classification of approaches per se, but answering the question of how non-knowledge has been represented formally in economic theory to date. This task requires, however, some detailed detection work, because nonknowledge has not been an explicit issue in economics yet.

Surely, there is knowledge economy, cf. Rooney et al (2008) [58], where knowledge is treated as a resource or a desirable asset. Also, knowledge is an important topic in information economics, as pioneered by Stigler

${ }^{*}$ E-mail: esvetlovaekarlshochschule.de

${ }^{\dagger}$ E-mail: hvanelstekarlshochschule.de 
(1961) [67], Akerlof (1970) [1], Spence (1973) [66], and Stiglitz (1975, 2002) [68, 69], where it is considered to be one of the tools to maximise profit. Generally, in economics, knowledge is considered as a good that is commonly available in principle (and should be used); the opposite — non-knowledge — is treated implicitly as a lack of information. In philosophy and the social sciences, the situation is not very different, though there are interesting recent attempts to overcome "theoretical preoccupations that underlie the study of knowledge accumulation," McGoey (2012) [49, p 1], and to develop an agenda for the social and cultural study of ignorance; cf. McGoey (2012) [49] and Proctor (2008) [56]. Ignorance should be treated "as more than "not yet known' or the steadily retreating frontier," Proctor (2008) [56, p 3], and should be separately accounted for as a strategic resource and the source of economic profit and progress; cf. Knight (1921) [45] and Esposito (2010) [26]. In economic theory, there have been occasional voices pleading for more attention to "true uncertainty", understood as the principle impossibility of foreseeing all future events that may occur in the exogenous world, cf. Davidson (1991) [14] and Dequech (2006) [17], and to "unknown unknowns", cf. Taleb (2007) [71] and Diebold et al (2010) [19]. However, non-knowledge has not become an independent issue of any significant interest or importance for economists so far. Thus, to find out how ignorance is formalised in the approaches considered here, we have to uncover first which aspects of decision-making are treated (often indirectly) as unknown, and which mathematical instruments are used to represent them.

Our focus is on the principle non-knowledge of future events in the exogenous world, which is the primary source of uncertainty. After providing, in Section 2, a brief historical overview to position the approaches considered within the ongoing debate on uncertainty, we are concerned with the formal mathematical representation of ambiguity in Section 3, and of unawareness in Section 4. Accordingly, we identify and review two approaches to the formalisation of non-knowledge in the literature: one based on economic agents' decisionmaking in the context of a state space representing the exogenous world, as in Savage's (1954) [61] axiomatisation and some successor concepts (ambiguity as situations with unknown probabilities), and one based on decision-making over a set of menus of potential future opportunities, providing the possibility of derivation of agents' subjective state spaces (unawareness as situation with imperfect subjective knowledge of all future events). Due to the large number of papers written on this topic, we have to be selective and, hence, cannot provide an exhaustive overview. We particularly draw attention to the last-mentioned line of research, namely uncertainty as unawareness, as it represents an exciting attempt to formalise "unknown unknowns" by radically departing from the mainstream paradigm of Savage's axiomatisation. Finally, in Section 5, we discuss the impending challenges and tasks of formalisation of non-knowledge in economics. We believe that without a detailed understanding of how non-knowledge has been represented in economics so far, no serious research agenda for studying ignorance as an independent part of economic theory can be developed. We hope that this article provides one of the first useful steps towards such an agenda.

\section{Historical developments}

Though there has not been an explicit discussion on non-knowledge in economic theory, this issue permanently turns up in relation to the topic of uncertainty. We identified three branches in the literature on decision-making of economic agents under conditions of uncertainty — risk, ambiguity and unawareness — and, in what follows, present those three directions and discuss the issue of knowledge versus ignorance in relation to each of them:

(i) risk: in formal representations, possible states and events regarding the exogenous world and their respective probabilities are known to all economic agents; they agree on the probability measure to be employed in calculations of individual utility,

(ii) uncertainty I - ambiguity: in formal representations, possible states and events are known but their respective probabilities are not known to the agents; each of them employs their own subjective (prior) probability measure in calculations of individual utility, 
(iii) uncertainty II - unawareness: in formal representations, possible states and events are known only incompletely to the agents; there is ignorance among them as regards relevant probability measures for calculations of individual utility.

This classification goes back to the work on uncertainty by Knight (1921) [45], Keynes (1921, 1937) [42, 43], Shackle $(1949,1955)$ [62, 63], and Hayek (1945) [37], who tightly connected the discussion of uncertainty with two kinds of knowledge, or rather ignorance: specifically, with imperfect knowledge of future events (uncertainty II), and with knowledge or non-knowledge of probability measures relating to future events (uncertainty I). Though the detailed depiction of the historical development of those concepts would go far beyond the scope of this paper, we consider it important to highlight the main ideas in this development in order to provide a topical frame for our discussion on the conceptualisation of non-knowledge in contemporary economic theory.

Generally, the authors mentioned differentiate between epistemological and ontological uncertainty. Epistemological uncertainty is related to situations where economic agents lack the knowledge necessary to construct adequate probability measures. According to Knight (1921) [45], e.g., theoretical, i.e., a priori probabilities on the one hand, and statistical probabilities on the other, are based on a valid fundament of knowledge: the law of large numbers, or statistical grouping. The a priori probability can be predicted using counting principles and a completely homogeneous classification of instances (e.g., by rolling dice), the statistical probability describes the frequency of an outcome based on a classification of empirical events or instances, given repeated trials. Knowledge is understood in both cases as (empirical) information that allows for the classification of possible outcomes. These two kinds of probability (a priori and statistical) can be measured, and in this sense are known and unanimously agreed upon by all agents involved in decision-making processes (the situation of risk). Hence, such probability measures can be reasonably referred to as objective.

However, Knight suggests that these two categories do not exhaust all possibilities for defining a probability measure; he adds “estimates", or subjective probabilities. Quoting Knight (1921) [45, p 225]: "The distinction here is that there is no valid basis of any kind for classifying instances. This form of probability is involved in the greatest logical difficulties of all ..." Knight refers to this last situation as a situation of uncertainty (ibid [45, p 233]); uncertainty can be defined as absence of probable knowledge. In the situation of risk, probabilities represent the measurable degree of non-knowledge; in the uncertainty situation, this degree is immeasurable, and in this sense probabilities are not known. Keynes (1921) [42] also suggested a concept of immeasurable probabilities as logical relationships, and argued in his 1937 paper — in unison with Knight — that economic agents lack a valid basis to devise probability measures. In his definition uncertainty exists, e.g., in the case of predicting the price of copper or the interest rate 20 years hence (Keynes (1937) [43, p 113]): "About these matters there is no scientific basis on which to form any calculable probability whatever. We simply do not know." Probabilities are used by economic agents as a convention that enables them to act (ibid [43, p 114]); at the same time, though probabilities are widely applied, they represent the agents' ignorance rather than their (scientific) knowledge.

Interestingly, in the later literature this issue was taken up by Ellsberg (1961) [21], who, in his experiments, distinguished between situations with known probability measures over some event space (when the color and number of the balls in an urn are known to agents; thus, they can form probabilities), and situations with unknown probability measures (agents know only the colors of balls but not the exact number of balls of each color; thus, they deal with the ignorance of probability). Ellsberg demonstrated empirically that people tend to prefer situations with known probability measures over situations with unknown probability measures; he explicitly referred to situations with unknown probability measures as ambiguous and named the phenomenon of avoiding such situations "ambiguity aversion" (corresponding to the term "uncertainty aversion" coined by Knight (1921) [45]).

It must be noted that the discussion about measurability of probabilities in economic life, as well as about their objective vs subjective character, was severely influenced and pulled in one particular, for a long time uncontested, direction by the line of argumentation due to Ramsey (1931) [57], de Finetti (1937) [27], and Savage (1954) [61]. Ramsey and de Finetti reacted to Knight's and Keynes' concepts of uncertainty as situations with 
immeasurable probabilities with the axiomatisation of subjective probabilities: they demonstrated that subjective probabilities can always be derived from the observed betting behaviour of economic agents, rendering the whole discussion about measurability and objectivity of probabilities seemingly obsolete. Adopting these results, Savage generalised the theory of decision under risk, i.e., the expected utility theory as conceived of originally by Bernoulli (1738) [4] and von Neumann and Morgenstern (1944) [53]. While the expected utility concept as an element of risk theory was based on objective probability measures, Savage combined expected utility theory and the subjective probability approach of Ramsey and de Finetti to deliver a new variant of an axiomatisation of decision under conditions of uncertainty - subjective expected utility theory. This concept was perfectly compatible with the Bayes-Laplace approach to probability theory and statistics where subjective prior probabilities can always be assumed to exist and adjusted in the process of learning. The crucial feature of Savage's probabilistic sophistication is the principle neglect of the Knightian distinction between risk and uncertainty, as Savage's concept presupposes that even if an objective probability measure for future events is not known, it can always be assumed that economic agents behave as if they apply an individual subjective (prior) probability measure to estimating the likelihood of future events; and these probability measures can in principle be derived a posteriori from an axiomatic model on the basis of empirical data on agents' choice behaviour. By this theoretical move, the immeasurability (and thus the knowability) issue is eliminated. The question of the validity of the subjective degrees of beliefs foundation, or of the origin of subjective probabilities, is beyond Savage's model, as these are built into the as-if-construction from the outset.

However, the Knightian distinction continued to bother economists and — especially after Ellsberg's (1961) [21] paper - a new branch of research appeared in the literature that endeavoured to re-introduce uncertainty, understood as absence of perfect knowledge of relevant probability measures, into economic theory. The most prominent attempt was delivered by Gilboa and Schmeidler (1989) [30]. In the next section, we will introduce the basic elements of their axiomatisation of decision under uncertainty in terms of non-unique probability measures, and contemplate how non-knowledge is represented in this concept. At the same time, the attentive reading of Knight, Keynes and Shackle suggests that the issue of uncertainty is not restricted to the question whether probabilities can be meaningfully defined or measured. There is a more fundamental issue of ontological uncertainty which is concerned with the principle unknowability of what is going on in an economic system; it goes beyond the scope of epistemic uncertainty.

Note that in the framework of epistemic uncertainty, knowledge that is relevant for the derivation of a meaningful probability measure is generally treated as information; compare the respective definition by Epstein and Wang (1994) [22, p 283], who define risk as a situation "where probabilities are available to guide choice, and uncertainty, where information is too imprecise to be summarized adequately by probabilities." It is interesting that also beyond the borders of economic theory — in the IPCC (2007) [41] report — the Knightian distinction between risk and uncertainty is understood as an epistemic one: "The fundamental distinction between 'risk' and 'uncertainty' is as introduced by economist Frank Knight (1921), that risk refers to cases for which the probability of outcomes can be ascertained through well-established theories with reliable complete data, while uncertainty refers to situations in which the appropriate data might be fragmentary or unavailable." (...) The clear relation "information (empirical data) - probabilities" is presupposed. The lack of knowledge, in this case, can be theoretically removed by becoming more skillful in calculating, or by collecting more information.

However, it should be stressed that Knight (as well as Keynes and Shackle) did not conceive of ignorance as lack of information but rather as ontological indeterminacy, the "inherent unknowability in the factors", see Knight (1921) [45, p 219]. Shackle (1955) [63] relates the genuinely imperfect knowledge about future events to the absence of an exhaustive list of possible consequences of choices. Traditional probability theory assumes that the list of consequences over which probability is distributed is an exhaustive list of possible outcomes, or, in Shackle's terms, hypotheses. However, so Shackle, if there is a residual hypothesis, that is, the list of possible consequences is incomplete, the probability model runs into trouble. By adding a hypothesis to the list of possible hypotheses, each corresponding probability of the previously known hypotheses has to be revised downwards; see Shackle (1955) [63, p 27]. If five possible hypotheses are considered and a sixth hypothesis is added, and additivity of probabilities is assumed, the probability of each of the initial five 
hypotheses is subsequently lower. This objection applies to both approaches, namely the frequentist approach to probability theory on the one hand, and the Bayes-Laplace approach which deals with belief-type subjective (prior) probability measures on the other, because neither can incorporate a residual hypothesis, or the principle non-knowledge of future states. Thus, referring to the genuinely imperfect knowledge about future events, Shackle (but also Knight and Keynes) expressed doubts whether probability theory in general is sufficient to account for decision under uncertainty, and whether it should be the central issue after all.

By far more important than the issue of devising suitable probability measures seems to be the nonknowledge of possible future states of the exogenous world and of related outcomes. Only if we manage to account properly for this imperfect knowledge, can we conceptualise properly human decision-making, or, in the words of Shackle (1959) [64, p 291], a non-empty decision. Crocco (2002) [11] explains: "An empty decision is the mere account of a formal solution to a formal problem. It is that situation where a person has a complete and certain knowledge about all possible choices and all possible outcomes of each choice. It is a mechanical and inevitable action," or, in the words of Heinz von Förster (1993) [28, p 153], every decidable (or perfectly known) problem is already decided; true decisions always presuppose genuine undecidability. In this sense, Savage's concept is rather concerned with empty decisions, because it presupposes situations with full knowledge of possible events, acts and outcomes, rendering agents' choices just a mechanical application of the personal utility-maximisation rule.

In economics, genuine undecidability should enter theory. Most economic decisions are truly undecidable because they take place under conditions of imperfect knowledge of the situation to be faced, which is in the sense of the American pragmatist philosopher John Dewey (1915) [18, p 506] a genuinely "incomplete situation": "something is 'there', but what is there does not constitute the entire objective situation." This "means that the decision-maker does not have complete knowledge of the following: (a) the genesis of the present situation, (b) the present situation itself, or (c) the future outcomes that remain contingent on the decisions that are made in the present situation;" see Nash (2003) [51, p 259]. According to Dewey (1915) [18], the situation is underdetermined, unfinished, or not wholly given.

This principle non-knowledge can be explained, so Shackle $(1949,1955)$ [62, 63], by the character of economic decisions, which he considers to be non-devisible, non-seriable, and crucial experiments. Non-devisible experiments imply only a single trial; non-seriable experiments are not statistically important even in the aggregate; an example of a seriable experiment is fire insurance: although no reasonable probability can be assigned to an individual house to burn down, if there are sufficiently many events, a (statistical) probability will emerge. Most importantly, economic decisions are crucial experiments: they inevitably alter the conditions under which they were performed (this definition applies to all strategic situations, e.g., chess play, but also financial markets). Within the genuinely social context of economic life, economic events are rather endogenous to the decision processes of agents and are dependent on the actions and thinking of other market participants. There are path dependencies and reflexivity; cf. Soros (1998) [65]. In general, a meaningful approach to decisionmaking should take into account that the future is principally unknowable, due to ontological features of the exogenous world such as openness, organic unity, and underdeterminacy. These are features which are typically attributed to complex systems; cf. Keynes et al (1926) [44, p 150]: "We are faced at every turn with the problems of Organic Unity, of Discreteness, of Discontinuity - the whole is not equal to the sum of the parts, comparisons of quantity fail us, small changes produce large effects, the assumptions of a uniform and homogeneous continuum are not satisfied." In such a system, not all constituent variables and structural relationships connecting them are known or knowable. Thus, in an open and organic system, some information is not available at the time of decision-making, and cannot be searched, obtained or processed in principle. Surprises, or unforeseen events, are normal, not exceptional. The list of possible events or states is not predetermined and very little, or nothing at all, can be known about the adequate probability measure for this radically incomplete set of future events.

These considerations require a more sophisticated distinction of decision-making configurations, namely a distinction that goes beyond the usual risk vs uncertainty as ambiguity debate. As Dequech (2006) [17, p 112] puts it: "Even though the decision-maker under ambiguity does not know with full reliability the probability 
that each event (or state of the world) will obtain, he/she usually knows all the possible event .... Fundamental uncertainty, in contrast, is characterized by the possibility of creativity and non-predetermined structural change. The list of possible events is not predetermined or knowable ex ante, as the future is yet to be created." What Dequech calls "fundamental uncertainty" (or "true uncertainty" in terms of some post-Keynesians (e.g., Davidson (1991) [14]) enters the recent debate in the economic literature under the label of "unawareness".

The unawareness concept, as introduced by Kreps 1979 [46], Dekel et al (1998, 2001) [15, 16], and Epstein et al (2007) [23], presupposes a coarse (imperfect) subjective knowledge of all possible future events. This concept criticises Savage's (1954) [61] axiomatisation and suggests a radical departure from it. Savage's axiomatisation is characterised by the in principle observability and knowability of all possible future events. These events belong to the primitives of the model and are assumed to be exogenous and known to all economic agents. In Savage's model, the (compact) state space representing the exogenous world the agents are continually interacting with is "a space of mutually exclusive and exhaustive states of nature, representing all possible alternative unfoldings of the world"; see Machina (2003) [48, p 26]. The exhaustiveness criterion is very restrictive and basically precludes non-knowledge of future states on the part of the agents. Machina (2003) [48, p 31] continues: "When the decision maker has reason to 'expect the unexpected' [or the residual hypothesis in terms of Shackle - the authors], the exhaustivity requirement cannot necessarily be achieved, and the best one can do is specify a final, catch-all state, with a label like 'none of the above', and a very ill-defined consequence." Obviously, true uncertainty as imperfect knowledge of possible future states of the exogenous world is not an element of Savage's model. The pioneers of the unawareness concept depart from Savage's axiomatisation by replacing the state space in the list of primitives by a set of menus over actions which are the objects of choice. This theoretical move allows for dealing with unforeseen contingencies, i.e., an inability of economic agents to list all possible future states of the exogenous world.

We now turn to give a more formal presentation of the two main concepts of uncertainty we discussed so far: uncertainty as ambiguity and uncertainty as unawareness.

\section{Uncertainty as ambiguity: non-knowledge of probability measures}

All decision-theoretical approaches to modelling an economic agent's state of knowledge regarding future developments of the exogenous world, the ensuing prospects for an individual's opportunities, and the agent's consequential choice behaviour under conditions of uncertainty employ an axiomatic description of the characteristic properties of observable choice behaviour and derive a quantitative representation of an agent's preferences in decision-making. Uncertainty in this context is generally interpreted as ambiguity perceived by an agent with respect to unknown probabilities by which future states of the exogenous world will be realised. In these approaches the standard assumption of neoclassical economics of an agent whose choices are fully rational is being maintained. The main issue of modelling here is to put forward a set of primitives which can be observed in principle in real-life settings, as well as a minimal set of axioms describing exhaustively the interconnections between these primitives, to provide the conceptual basis for (in general highly technically demanding) mathematical proofs of representation theorems. Most approaches in the literature propose an expected utility (EU) representation of an agent's preferences in terms of a real-valued personal utility function which is an unobservable theoretical construct, thus following the quantitative game-theoretical tradition of von Neumann and Morgenstern (1944) [53]. A related issue is the question to what extent an agent's choice behaviour can be reasonably viewed as influenced by a set of personal subjective probabilities regarding the (unknown) future states of the exogenous world. We begin by briefly reviewing the central aspects of the axiomatic approach taken by Savage (1954) [61] to describe one-shot choice situations - the subjective expected utility (SEU) framework, which attained the prominent status of a standard model in decision theory.

The primitives in Savage (1954) [61] are

(i) an exhaustive set of mutually exclusive future states $\omega$ of the exogenous world which an agent cannot actively take an influence on; these constitute a state space $\Omega$ which is assumed to be continuous, com- 
pact, and can be partitioned into a finite number of pairwise disjoint events; possible events $A, B, \ldots$ are considered subsets of $\Omega$, with $2^{\Omega}$ the set of all such subsets of $\Omega$,

(ii) a finite or infinite set of outcomes $x$ contingent on future states $\omega$, forming an outcome space $\boldsymbol{X}$, and

(iii) a weak binary preference order $\succeq$ ("prefers at least as much as") defined over the agent's objects of choice - a set of potential individual acts $f$ an agent may consciously take in reaction to realised future states $\omega$ of the exogenous world, yielding predetermined outcomes $x-$, describing their personal ranking of available options; these acts form a space $\boldsymbol{F}$.

In more detail, an act is defined as a (not necessarily real-valued, continuous) mapping $f: \Omega \rightarrow \boldsymbol{X}$ from the set of future states $\Omega$ to the set of possible outcomes $\boldsymbol{X}$, so the set of acts available to an agent at a given instant in time, in view of known future states $\omega$ but of unknown probabilities, is $\boldsymbol{F}=\boldsymbol{X}^{\boldsymbol{\Omega}}$. There is no additional structure needed in this model regarding measures or topology on either space $\Omega$ or $\boldsymbol{X}$, except for continuity and compactness of $\boldsymbol{\Omega}$. An observable weak binary preference order over the set of acts is given by $\succeq \subset \boldsymbol{F} \times \boldsymbol{F}$, intended to reflect an agent's subjective beliefs regarding future states $\omega$, and the usefulness of acts the agent may take in response to ensuing states.

Savage introduces a minimal set of seven axioms (P1 to P7) to characterise the theoretical nature of this preference order over acts (and, by implication, related outcomes), which are commonly referred to in the literature as weak order resp. completeness, sure-thing principle, state-independence, comparative probability, non-triviality, Archimedean, and finitely additive probability measures; cf. Nehring (1999) [52, p 105] and Gilboa (2009) [29, p 97ff]. These axioms constitute the foundation of a representation theorem proved by Savage which states that an agent's (one-shot) choice behaviour under conditions of uncertainty may be viewed as if it was guided by (i) a real-valued personal utility function $U: X \rightarrow \mathbb{R}$ that assigns subjective value to specific outcomes $x \in \boldsymbol{X}$, and (ii) a single finitely additive subjective probability measure $\mu: 2^{\Omega} \rightarrow[0,1]$ on the space of all possible future events $2^{\Omega}$. In particular, an agent's choice behaviour may be modelled as if for the acts $f$ available to them they strive to maximise a real-valued EU preference function $V: \boldsymbol{F} \rightarrow \mathbb{R}$, defined by

$$
V(f):=\int_{\Omega} U(f(\omega)) \mu(\mathrm{d} \omega) .
$$

Hence, in this setting an act $f \in \boldsymbol{F}$ is weakly preferred by an agent to an act $g \in \boldsymbol{F}$, iff $V(f) \geq V(g)$.

The elements of Savage's SEU model may be schematically summarised in terms of a decision matrix of the following structure (here for a partition of the continuous and compact $\Omega$ into a finite number $n$ of pairwise disjoint events):

\begin{tabular}{c|cccc|l} 
probability measure $\mu$ & $P\left(\omega_{1}\right)$ & $P\left(\omega_{2}\right)$ & $\ldots$ & $P\left(\omega_{n}\right)$ & \\
\hline acts $\boldsymbol{F} \backslash$ states $\boldsymbol{\Omega}$ & $\omega_{1}$ & $\omega_{2}$ & $\ldots$ & $\omega_{n}$ & \\
\hline$f_{1}$ & $x_{11}$ & $x_{12}$ & $\ldots$ & $x_{1 n}$ & \\
$f_{2}$ & $x_{21}$ & $x_{22}$ & $\ldots$ & $x_{2 n}$ & outcomes $\boldsymbol{X}$ \\
$\vdots$ & $\vdots$ & $\vdots$ & $\ddots$ & $\vdots$ &
\end{tabular},

where $0 \leq P\left(\omega_{i}\right) \leq 1$ and $\sum_{i} P\left(\omega_{i}\right)=1$ (and generally: $\mu \geq 0$ and $\int_{\Omega} \mu(\mathrm{d} \omega)=1$ ). Note that, formally, Savage's framework reduces an agent's situation of decision under uncertainty, in the Knightian sense of not knowing the probability measure associated with $\left(\Omega, 2^{\Omega}\right)$ a priori, to a manageable situation of decision under risk by introducing a single subjective Bayesian prior probability measure as a substitute. This is to say, every single economic agent possesses for themselves a unique probability measure which they employ in their individual calculations of utility; a probability measure is thus known to every individual from the outset, but there is no reason whatsoever that these measures should coincide between agents.

Savage's main claim is that his framework can be used to explicitly derive for an arbitrary economic agent who makes rational choices in parallel (i) a unique subjective probability measure $\mu$ over $\left(\Omega, 2^{\Omega}\right.$ ), and (ii) a personal utility function $U$ over $\boldsymbol{F}$ (unique up to positive linear transformations), from observation of their choice 
behaviour in practice. For the sequel it is worth mentioning that Savage's numerical SEU representation (1) can be interpreted to fall into either of the categories of ordinal or additive EU representations.

Various authors have criticised Savage's SEU model for different reasons, where in particular the claim is that one or more of his axioms are regularly being violated in real-life situations of (one-shot) choice. Bewley $(1986,2002)$ [5, 6], for example, points the finger to the completeness axiom P1 in that he considers it unrealistic to assume that all agents have a clear-cut ranking of all the acts available to them, when it need not necessarily be clear from the outset which acts comprise the complete set $\boldsymbol{F}$. In his work he therefore proposes an axiomatic alternative to Savage's SEU model which discards the completeness axiom in favour of an inertia assumption regarding the status quo of an agent's personal situation.

More prominent still is Ellsberg's (1961)[21] empirical observation that in situations of choice under uncertainty rational agents need not necessarily act as subjective expected utility maximisers: given the choice between a game of chance with known probabilities of the possible outcomes and the identical game of chance where the probabilities are unknown, the majority of persons tested exhibited the phenomenon of uncertainty aversion by opting for the former game. Ellsberg showed that this kind of behaviour correspond to a violation of Savage's sure-thing principle axiom P2.

A possible resolution of this conflict was suggested in the multiple priors maxmin expected utility (MMEU) model due to Gilboa and Schmeidler (1989) [30], which takes uncertainty aversion explicitly into account by stating that under conditions of uncertainty an agent need not have to have a unique subjective prior probability measure $\mu$, but rather an entire set $\Pi$ worth of such measures $\pi$ from which they select in making decisions according to the maxmin principle. In this sense, Gilboa and Schmeidler take an explicit attempt at formalising Knightian uncertainty in problems of decision-making, interpreted as situations with in principle unknowable probability measures over $\left(\boldsymbol{\Omega}, 2^{\boldsymbol{\Omega}}\right)$. The degree of an agent's ignorance is encoded in the generically unconstrained cardinality of the set of Bayesian priors $\Pi$ : no criteria are formulated according to which an agent assesses the relevance of any particular probability measure that is conceivable for a given situation of decision-making. Non-knowledge regarding the likelihood of future events here is linked to the number of elements included in the individual set $\Pi$ that is employed in an agent's individual calculation of utility and so is represented in a more comprehensible fashion than in Savage's framework.

Nevertheless, the primitives of the MMEU model are unchanged with respect to Savage's SEU model. Based on a minimal set of six axioms (A1 to A6) referred to resp. as weak order, certainty-independence, continuity, monotonicity, uncertainty aversion and non-degeneracy, the representation theorem Gilboa and Schmeidler (1989) [30] prove employs a real-valued preference function $V: \boldsymbol{F} \rightarrow \mathbb{R}$ defined by the minimum expected utility relation

$$
V(f):=\min _{\pi \in \Pi} \int_{\Omega}\left(E_{f(\omega)} U\right) \mathrm{d} \pi,
$$

with $\Pi \subset \Delta(\boldsymbol{\Omega})$ a non-empty, closed and convex set of finitely additive probability measures over $\left(\boldsymbol{\Omega}, 2^{\boldsymbol{\Omega}}\right)$, and $U: \boldsymbol{X} \rightarrow \mathbb{R}$ a non-constant real-valued personal utility function. Again, an act $f \in \boldsymbol{F}$ is then weakly preferred by an agent to an act $g \in \boldsymbol{F}$, iff $V(f) \geq V(g)$.

Since its inception, Gilboa and Schmeidler's MMEU model has enjoyed a number of applications in the econometrical literature; e.g. in Epstein and Wang (1994) [22] on intertemporal asset pricing; Hansen et al (1999) [33] on savings behaviour; Hansen and Sargent (2001, 2003) [34, 35] on macroeconomic situations; Nishimura and Ozaki (2004) [54] on a job search model; and Epstein and Schneider (2010) [24] on implications for portfolio choice and asset pricing. Rigotti and Shannon (2005) [59], who propose an approach to formalising uncertainty in financial markets on the basis of Bewley's $(1986,2002)[5,6]$ idea of discarding Savage's completeness axiom P1, contrast their findings on the impact of uncertainty on equilibrium configurations in decision-making processes with corresponding consequences arising from an MMEU perspective.

The strongest criticism to date of Savage-type state space models of decision-making under conditions of uncertainty was voiced at the end of the 1990ies by Dekel et al (1998) [15]. They showed that given one considers it unrealistic for an economic agent to be aware of all possible future states $\omega$ of the exogenous world, 
a standard state space model is incapable of consistently incorporating the dimension of an agent's unawareness of future contingencies. The basis of the formal treatment of the issue at hand are information structures referred to as possibility correspondences. A possibility correspondence amounts to a function $P: \Omega \rightarrow 2^{\Omega}$ that maps elements $\omega$ in some state space $\Omega$ to subsets thereof, so that $P(\omega)$ is interpreted as the set of states an agent considers possible when the realised state is $\omega$. In this picture, an agent "knows" an event $E \in 2^{\Omega}$ at a state $\omega$ provided $P(\omega) \subseteq E$. Hence, given a possibility correspondence $P$, a knowledge operator $K: 2^{\Omega} \rightarrow 2^{\Omega}$ is determined by

$$
K(E):=\{\omega \in \Omega \mid P(\omega) \subseteq E\} \quad \text { for all } E \in 2^{\Omega} ;
$$

$K(E)$ represents the set of states in $\Omega$ for which an agent knows that event $E$ must have occurred. According to Dekel et al, it is commonplace to assume that such a knowledge operator features the properties of (i) necessitation, meaning $K(\boldsymbol{\Omega})=\boldsymbol{\Omega}$, and (ii) monotonicity, meaning $E \subseteq F \Rightarrow K(E) \subseteq K(F)$. In addition, an unawareness operator may be defined as a mapping $U: 2^{\Omega} \rightarrow 2^{\Omega}$, so that $U(E)$ is to be regarded as the set of states in $\Omega$ where an agent is unaware of the possibility that event $E$ may occur. With these structures in place, a standard state space model is represented by a triplet $(\Omega, K, U)$.

To obtain their central result, Dekel et al require a minimal set of only three axioms which characterise the nature of the operators $K$ and $U$ : these demand that for every event $E \in 2^{\Omega}$, (i) $U(E) \subseteq \neg K(E) \cap$ $\neg K\left(\neg K(E)\right.$ ), called plausibility, ${ }^{1}$ (ii) $K(U(E))=\emptyset$, called KU introspection, and (iii) $U(E) \subseteq U(U(E)$ ), called AU introspection. Given a standard state space model $(\Omega, K, U)$ satisfies these three axioms, the theorem proven by Dekel et al (1998) [15, p 166] states that in such a setting (a) "the agent is never unaware of anything," provided $K$ satisfies the necessitation property, and (b) "if the agent is unaware of anything, he knows nothing," provided $K$ satisfies the monotonicity property. This result renders standard state space models void as regards the intention of formally capturing an agent's unawareness of subjective contingencies in a non-trivial way.

The work by Dekel et al (1998) [15], in particular, triggered a series of papers written during the last decade, which aspire to include an agent's unawareness of future subjective contingencies in a coherent model that continues to employ a kind of EU representation of an agent's manifested preferences in situations of choice under conditions of uncertainty. We turn to highlight the, in our view, most important papers of this development next.

\section{Uncertainty as unawareness: non-knowledge of complete state spaces}

Since the status of possible future states $\omega$ of the exogenous world as a primitive in a decision-theoretical model on an agent's choice behaviour under conditions of uncertainty is questionable due to the lack of a convincing operational instruction for observation of such states, a number of authors have dropped the state space $\Omega$ from the set of primitives altogether and turned to focus instead on the description of an agent's preferences when they are unaware of some future subjective contingencies which take a direct influence on future outcomes such as the pay-offs of certain actions. In the papers to be considered in the following, the conceptual line of thought pursued in which originated in the work by Kreps (1979) [46], the primitives underlying this alternative approach comprise in general

(i) a (typically finite) set $\boldsymbol{B}$ of alternative opportunities, actions, or options; a generic element in this set will be denoted by $b$,

(ii) a (typically finite) set $\boldsymbol{X}$ of all conceivable non-trivial menus compiled from elements in $\boldsymbol{B}$, with a generic element denoted by $x$; note that $\boldsymbol{X}=2^{\boldsymbol{B}} \backslash\{\emptyset\}$,

(iii) a weak binary preference order $\succeq$ defined over the agent's objects of choice, presently menus in $\boldsymbol{X}$.

\footnotetext{
${ }^{1}$ The symbol $\neg$ denotes complementation.
} 
The setting conceived of in this approach considers a two-stage choice process in which an agent will initially ("now") choose a particular menu $x$, from which, contingent on subsequently ensuing states $\omega$ of the exogenous world, they will choose a specific element $b$ at an unmodelled later stage ("then"). ${ }^{2}$ Hence, two kinds of (weak) binary preference orders need to be introduced: an "ex ante preference" (preference "now") over the set $\boldsymbol{X}, \succeq \subset \boldsymbol{X} \times \boldsymbol{X}$, and an "ex post preference" (preference "then") over $\boldsymbol{B}$ contingent on a realised state $\omega$, $\succeq_{\omega}^{*} \subset \boldsymbol{B} \times \boldsymbol{B}$; cf. Dekel et al (2001) [16]. Generally, authors then proceed to formulate minimal sets of axioms for the ex ante preference order $\succeq$, on the basis of which they prove representation theorems for modelling an agent's choice behaviour under conditions of uncertainty in the sense that the agent is unaware of some future subjective contingencies. A particularly interesting feature of some of the works to be discussed in the sequel is the possibility to derive in principle an agent's subjective state space regarding future subjective contingencies from observed choice behaviour, given some form of EU representation of the agent's preference relation is employed. This aspect is key to a meaningful representation of non-knowledge in economic theory. It is also seen as an intermediate step towards derivation of an agent's subjective probability measure regarding choice behaviour under conditions of uncertainty on the basis of empirical data.

Kreps (1979) [46], in his pioneering paper, considers an agent with a "desire for flexibility" as regards decision-making, the choice behaviour of which, however, may not satisfy "revealed preference". He formalises these properties of an agent's envisaged choice behaviour in terms of the following two axioms: for all $x, x^{\prime}, x^{\prime \prime} \in \boldsymbol{X}$,

$$
x \supseteq x^{\prime} \quad \Rightarrow \quad x \succeq x^{\prime}
$$

and

$$
x \sim x \cup x^{\prime} \quad \Rightarrow \quad x \cup x^{\prime \prime} \sim x \cup x^{\prime} \cup x^{\prime \prime},
$$

with $\sim$ denoting the indifference relation on $\boldsymbol{X}$. Note that in the literature the axiom (5) is often referred to as the monotonicity axiom. Kreps, in his discussion, does not make explicit an agent's uncertainty regarding unawareness of (some) future subjective contingencies. Rather, it is implied by the agent's "desire for flexibility". He continues to prove that, given a "dominance relation" on $\boldsymbol{X}$ defined by

$$
x \geq x^{\prime} \quad \text { if } \quad x \sim x \cup x^{\prime},
$$

and the axioms stated before, an agent's preferences on $\boldsymbol{X}$ can be sensibly described as if they were "maximizing a "state dependent utility function of subsequent consumption"" in terms of a formal real-valued preference function $V: X \rightarrow \mathbb{R}$, defined by

$$
V(x):=\sum_{s \in \boldsymbol{S}} \max _{b \in x} U(b, s) .
$$

Here $S$ denotes the unobservable finite subjective state space of an agent's personal tastes, with generic element $s$, and $U: \boldsymbol{B} \times \boldsymbol{S} \rightarrow \mathbb{R}$ is the agent's unobservable state-dependent real-valued utility function of alternative opportunities available in the finite set $\boldsymbol{B}$. Kreps points out that this representation is principally ordinal in character. The bottom-line of Kreps' approach is that the set of state-dependent ex post utilities $\{U(\cdot, s) \mid s \in$ $\boldsymbol{S}\}$, expressing the agent's beliefs on potential future pay-offs, can be interpreted as an agent's implicitly given coherent subjective state space which describes their uncertainty regarding ex post choices over the set $\boldsymbol{B}$, and so can be legitimately used as a model of unforeseen contingencies (cf. Kreps (1992) [47]).

However, as Dekel et al (2001) [16, p 892, p 896f] emphasise, Kreps' implied subjective state space $\{U(\cdot, s) \mid s \in \boldsymbol{S}\}$ of an agent is far from being determined uniquely, since the axioms he proposed prove not to be sufficiently restrictive for this purpose. It is this feature in particular, which these authors set out to overcome in their own work. To accomplish this goal, Dekel et al (2001) [16] extend Kreps' analysis in two respects. On the one-hand side, here the agent's objects of choice are, in the spirit of von Neumann and Morgenstern (1944)

\footnotetext{
${ }^{2}$ As will be described in the following, in some of the works to be reviewed the elements of choice at stage "then" can be more complex objects than simply elements $b \in \boldsymbol{B}$.
} 
[53], sets of lotteries $\Delta(\boldsymbol{B})$ defined over finite sets of future alternative opportunities $\boldsymbol{B}$, on the other, the assumption of an agent's strict preference for flexibility is relaxed to also allow for a preference for commitment in instances when this appears valuable. The latter feature introduces the possibility of an agent's view "ex ante" to differ from their view "ex post". To continue with the primitives: Dekel et al take the set $\Delta(B)$ to correspond to a set of probability measures over $\boldsymbol{B}$; a generic lottery in $\Delta(\boldsymbol{B})$ is denoted by $\beta$. Subsets of $\Delta(\boldsymbol{B})$ are referred to as menus $x$, with $\boldsymbol{X}$ denoting the set of all non-empty subsets of $\Delta(\boldsymbol{B}) . \boldsymbol{X}$ is endowed with a Hausdorff topology and constitutes the formal basis of an agent's binary ex ante preference order, $\succeq \subset \boldsymbol{X} \times \boldsymbol{X}$. The two-stage choice process of Kreps (1979) [46] remains qualitatively unchanged: the agent chooses a menu $x \in \boldsymbol{X}$ "now", and a lottery $\beta \in x$ "then".

Dekel et al's different kinds of representations of an agent's ex ante preference order $\succeq$ over menus $x$ of lotteries correspond to triplets $(\Omega, U, u)$, comprising the following three common elements: a non-empty (exogenous) state space $\Omega$ serving merely as an index set to label ex post preferences over $\Delta(\boldsymbol{B})$, a statedependent real-valued personal utility function $U: \Delta(\boldsymbol{B}) \times \Omega \rightarrow \mathbb{R}$, and a real-valued personal aggregator function $u: \mathbb{R}^{\Omega} \rightarrow \mathbb{R}$. The aggregator function is a rather special feature in Dekel et al's analysis. It is given the role of translating an agent's ex post utility levels of menus $x$ into corresponding ex ante values, making the strong assumption that, in the model proposed, an agent has a coherent view of all future utility possibilities of menus $x$ available to them. The ex post preference order $\succeq_{\omega}^{*}$ over $\Delta(\boldsymbol{B})$, given a state $\omega \in \boldsymbol{\Omega}$, can be viewed as being encoded in the utility function $U(\cdot, \omega)$. In consequence, Dekel et al define an agent's subjective state space as the set $\boldsymbol{P}(\boldsymbol{\Omega}, U):=\{U(\cdot, \omega) \mid \omega \in \boldsymbol{\Omega}\}$.

On the basis of an ex ante preference-characterising minimal set of seven axioms (A1 to A7), referred to as weak order, continuity, non-triviality, indifference to randomisation (IR), independence, weak independence and monotonicity, resp., Dekel et al (2001) [16] prove existence theorems for three kinds of EU representations, all of which can be cast in the form of a real-valued preference function $V: X \rightarrow \mathbb{R}$ defined by

$$
V(x):=u\left(\left(\sup _{\beta \in x} U(\beta, \omega)\right)_{\omega \in \boldsymbol{\Omega}}\right),
$$

with $U(\cdot, \omega)$ an EU affine function in line with von Neumann and Morgenstern (1944) [53], i.e., for all $\beta \in$ $\Delta(\boldsymbol{B})$ and $\omega \in \boldsymbol{\Omega}$,

$$
U(\beta, \omega):=\sum_{b \in \boldsymbol{B}} \beta(b) U(b, \omega) .
$$

The main results following from the proofs of the EU representation theorems for the binary ex ante preference order $\succeq$ are: (i) uniqueness of an agent's subjective state space $\boldsymbol{P}(\Omega, U)$ related to their binary ex post preference order, as well as essential uniqueness of the associated aggregator function $u$, (ii) the size of an agent's subjective state space $\boldsymbol{P}(\Omega, U)$ can be interpreted as a measure of their uncertainty about future subjective contingencies, while the associated aggregator $u$ indicates whether such contingencies trigger a preference for commitment or rather for flexibility, (iii) ordinal EU representations offer the smallest subjective state space $\boldsymbol{P}(\Omega, U)$ possible for any ordinal representation, and (iv) existence of an additive EU representation when in particular the standard independence axiom due to von Neumann and Morgenstern (1944) [53] holds; the former is given by a real-valued preference function $V: X \rightarrow \mathbb{R}$ such that (up to monotone transformations)

$$
V(x)=\int_{\Omega} \sup _{\beta \in x} U(\beta, \omega) \mu(\mathrm{d} \omega),
$$

with $\mu$ a (non-unique) finitely additive probability measure on $\left(\boldsymbol{\Omega}, 2^{\boldsymbol{\Omega}}\right)$. This last result, providing a representation in line with "standard" approaches, raises ideas on the possibility of identification of an agent's probability measure over their subjective state space $\boldsymbol{P}(\Omega, U)$, analogous to one of the central outcomes of Savage's (1954) [61] SEU model. However, it is the state-dependence of an agent's ex post preference which renders this objective currently quite unrealistic. 
Dekel et al's (2001) [16, p 894] approach contains an inherent interpretational difficulty, which these authors briefly address: the model represents an agent with an at least partially incomplete concept of future subjective contingencies "now" with an agent with complete knowledge of all utility possibilities of menus "then"; does the model, nevertheless, deal consistently with an agent's non-knowledge of (some) future subjective contingencies? Dekel et al do not see a need for full commitment to this issue, but leave this point by resorting to the idea of an "as if" representation of their model. However, to put their model to the test, they call for the identification of a concrete Ellsberg-type example of an agent's choice behaviour which is in contradiction with (some of) their axioms; cf. Dekel et al (2001) [16, p 920].

This challenge was met in the work by Epstein et al (2007) [23], in which they focus on criticising Dekel et al's additive EU representation in particular. The main argument Epstein et al give states that an economic agent who is aware of their incomplete knowledge of future subjective contingencies, and in particular is averse to this personal state of affairs, will feel a need to hedge against this uncertainty by randomisation over options available to them, thus providing a case of violation of Dekel et al's independence axiom. In addition, these authors argue that the impossibility of fully describing all future contingencies relevant to an agent may lead to the failure of quantifying an agent's uncertainty about utilities "then" in terms of just a single probability measure, as Dekel et al do in their additive EU representation. In Epstein et al's (2007) [23, p 359] view, Dekel et al's model therefore precludes a consistent representation of incompletely known future subjective contingencies and ambiguity about an agent's preferences "then".

To overcome the conceptual problems of Dekel et al's model — in particular, to capture the ambiguity due to an agent's incomplete knowledge of future subjective contingencies, and their induced tendency for hedging against it - Epstein et al (2007) [23] propose two alternative axiomatic models of an agent's ex ante choice behaviour. These can be considered modifications of Dekel et al's approach in the following sense. The first model maintains the assumption of the IR axiom to hold, while the independence axiom is being relaxed; in the second model both the IR and the independence axioms are dropped, and the primitives of the model are extended to include random menus. The two models exhibit a qualitative difference as regards the status of ex post ambiguity that an agent finds themself exposed to "then". In the first model, an agent "now" expects to gain complete knowledge "then" of a state realised in the meantime, i.e., before they choose a lottery $\beta$ from the ex-ante-preferred menu $x$; hence, ex post ambiguity is resolved. However, "now" the agent is uncertain about their actual preferences "then". In the second model, on the other hand, an agent "now" reckons that even "then" their knowledge of all relevant contingencies will remain incomplete, leaving their preferences "then" somewhat vague due to the lack of a complete view of all of the options available to them. In the present work this circumstance is modelled in terms of a restricted set of utility functions (over lotteries) with unknown likelihoods. Ex post ambiguity persists, making hedging against uncertainty "then" (and related potentially unfavourable outcomes) a valuable tool.

In model I, Epstein et al (2007) [23] implement an agent's need for hedging by following the ideas of Gilboa and Schmeidler (1989) [30] on uncertainty aversion in that, via introducing a mixing operation defined over menus, an axiomatisation of a multiple-priors utility representation of an agent's ex ante preferences is proposed. Starting from a minimal set of eight axioms requiring (weak) order, monotonicity, IR, non-degeneracy, ${ }^{3}$ preference convexity, worst, certainty-independence, and mild continuity, the corresponding representation theorem states that an agent's ex ante choice behaviour amounts to maximising a real-valued preference functional $V_{M P}: \boldsymbol{X} \rightarrow \mathbb{R}$, given by

$$
V_{M P}(x):=\min _{\pi \in \Pi} \int_{\boldsymbol{N}} \max _{\beta \in x} U(\beta) \mathrm{d} \pi(U),
$$

with $\Pi$ a (non-unique) convex and compact set of Borel probability measures on the space $N$ of specifically normalised ex post utility functions; cf. Epstein et al (2007) [23, p 365].

For their model II, in order to provide a formal basis for dealing with persistent coarseness "then" of an agent's perception of future subjective contingencies, Epstein et al (2007) [23] enlarge the set of an agent's

\footnotetext{
${ }^{3}$ In the literature the names non-degeneracy and non-triviality are used synonymously for one of the axioms.
} 
objects of choice to also include random menus of lotteries. This thus yields a set of Borel probability measures $\Delta(\boldsymbol{X})$ defined over menus in $\boldsymbol{X}$. A generic element in $\Delta(\boldsymbol{X})$ is denoted by $P$. Proposing a minimal set of six axioms comprising (weak) order, continuity, non-degeneracy, first-stage independence, dominance, and certainty reversal of order to hold, a representation theorem is proved for an agent's binary ex ante preference order $\succeq$ over random menus in $\Delta(\boldsymbol{X})$ to the extent that, in this model, an agent's choice behaviour corresponds to maximising a real-valued preference functional $\mathcal{V}_{P C}: \Delta(\boldsymbol{X}) \rightarrow \mathbb{R}$, given by

$$
\mathcal{V}_{P C}(P):=\int_{\boldsymbol{X}}\left[\int_{\mathcal{K}^{c c}\left(\boldsymbol{N}^{*}\right)} \max _{\beta \in x} \min _{U \in \mathcal{U}} U(\beta) \mu(\mathrm{d} U)\right] \mathrm{d} P(x),
$$

with $\mu \in \Delta\left(\mathcal{K}^{c c}\left(\boldsymbol{N}^{*}\right)\right)$ a Borel probability measure over the set $\mathcal{K}^{c c}\left(N^{*}\right)$ of closed, convex and comprehensive Hausdorff-topology subsets of the compact space of specifically normalised ex post utility functions $N^{*}$ (cf. Epstein et al (2007) [23, p 366]), which is unique up to linear transformations. $\mathcal{U} \subset \boldsymbol{N}^{*}$ denotes the subset of normalised ex post utility functions conceived of by an agent "now", which, however, to them in that instance have unknown likelihoods as regards realisation "then". In this respect, $\boldsymbol{N}^{*} \backslash \mathcal{U}$ may be interpreted as relating to an agent's unawareness (or non-knowledge) "now" of possible subjective contingencies "then".

\section{Discussion and outlook}

Now, having discussed the state of the art in the literature, we should question if the formal representation of non-knowledge in economic theory has been satisfactory so far. Moreover, in what follows we sketch some promising directions of research which we did not address in detail in this paper.

As highlighted in section 2, true uncertainty and, thus, genuine non-knowledge about the future, are features of the situation in which an agent is unaware of all future contingencies, not (just) due to their limited ability to calculate, or to search for information, but due to the very nature of any economic system. The major insight of Knight, Keynes, Shackle, and some Post-Keynesians, was that economic systems are open and organic unities that are genuinely indeterminate; every decision situation is incomplete because it undergoes a constant change while people decide and act and, by doing so, influence the set of relevant variables; hence, the major characteristics of the decision situation - first of all, the future states that are possible and conceivable cannot be sufficiently determined; they are unknown.

We already mentioned the following concrete reasons for the indeterminacy of decision situations: (i) the big world issue (i.e., the indefinite, non-exhaustive, number of possible future states), (ii) the endogeneity of the decision situations, i.e., the dependence of future outcomes on decisions which are prepared and made in the present, and (iii) the social contingency which is typical for economic systems, where the indeterminacy increases due to the dependence of an agent's decisions on what other agents decide. Are those issues adequately reflected in the ambiguity and unawareness approaches, which we discussed in this paper?

\subsection{Big world issue}

Savage's (1954) [61] axiomatisation was often criticised for its restrictive assumption of the "small" world: the list of possible events is presupposed to be exhaustive (though Savage [61, p 16] himself referred to such an assumption as "preposterous"). Some of the follow-up concepts discussed in our paper differ in their treatment of this issue.

The uncertainty as ambiguity approaches we mentioned continue to employ Savage-type state spaces as primitives, which are continuous, compact, and can be partitioned into a finite number of mutually exclusive events, while there is an uncountable number of different states. Although in principle no additional structure is needed, some authors like Epstein and Wang (1994) [22, p 206] assume on the state space the existence of a metric and a particular ("weak convergence") topology, suggesting that one can construct an indefinite number of different subsets of the state space; the boundaries of such subsets are not entirely clear. The question arises 
if, in the end, such mathematical structures make everything possible and thinkable, thus offering a loophole for the assumption that the list of possible events is not exhaustive. It is worthwhile mentioning here that the formal handling, but even more so providing compelling interpretations, of a potential infinitude of possibilities or states regularly proves a delicate issue in most (if not all) areas of applied mathematics and statistics; see, e.g., Hawking and Ellis (1973) [36].

In the uncertainty as unawareness models, in contrast, the big world issue, which relates to the state space representing the exogenous world, is of less importance, since here the focus of the analyses is on an agent's subjective state space. This concept, however, does not belong to the set of the primitives of the theory. This issue is closely related to the exogeneity vs endogeneity topic which we turn to discuss next.

\subsection{Endogeneity of state space}

In our view, the question of Machina (2003) [48, p 18]: "Do individuals making choices under uncertainty face states of nature, or do they create them?" remains one of the most crucial and controversial in decision theory. In Savage's concept, the state space represents nature's exogenous states, i.e., their emergence cannot be influenced by agents' decisions and actions; an agent just observes the states and is not an active part of the decision situation.

In economics, as well as in the social sciences, however, there is increasing attention being payed to the issue of creation of economic reality by the actions and decisions of economic agents; e.g., one considers the notions of exogenous risk, performativity and reflexivity; cf. Danielsson and Shin (2003) [12], Danielson et al (2009) [13], Callon (1998) [9], MacKenzie (2006) [50], and Soros (1998) [65]. There is also an interesting movement in the direction of constructive decision theory, where decision-relevant states are not given but "constructed by the DM (decision maker) in the course of deliberating about questions such as 'How is choice A different from choice B?' and 'In what circumstances will choice A turn out better than choice B?"'; see Blume et al (2009) [7, p 1f].

Concerning the papers discussed in the article at hand, the works on uncertainty as ambiguity focus on the non-knowledge of probabilities; here, the state space remains exogenous. However, the unawareness literature makes an interesting and important move towards the conceptualisation of an endogenous state space: future outcomes ("then") are contingent on decisions made at present ("now"); the subjective state space is not directly observable but can be derived from the only variable observable: an agent's behaviour, or an agent's preferences (e.g., for flexibility).

However, an important question remains if, in the unawareness literature, we really deal with a truly endogenous state space, as understood in the concepts of endogenous risk, performativity and reflexivity. There is already raised some criticism in the literature, e.g. by Sagi (2006) [60], who is concerned about the static nature of the theoretical construction in the unawareness approaches: "The decision maker chooses among menus, uncertainty over her subjective states is assumed to resolve and then the decision maker selects from the menu. However, there is no explicit modelling of ex-post choice and no role for consistency between realized tastes and tastes inferred from ex-ante preferences", see Sagi (2006) [60, p 307]. We also think that the representation of true endogeneity - as a central determinant of non-knowledge — should be dynamic: uncertainty cannot be resolved, as a situation constantly changes, so that the ex-post choice should not be modelled as a mechanic, or empty (i.e., predetermined) decision. The other important dynamic aspect is the modelling of the evolution of the state space itself (how it expands and changes); the genesis of a decision situation should be taken into consideration. There are some interesting ideas aiming at these issues, e.g. by Hayashi (2012) [38], and Grant and Quiggin (2007) [32]. The latter authors model the notion of discovery of the principally unknown space states by decision-makers. We consider this issue to be crucial for making further progress in the unawareness and non-knowledge literature. 


\subsection{Social contingency}

The idea of an endogenous space state (as well as the notions of endogenous risk, performativity and reflexivity) goes beyond the subjective level of decision-making: future states are unknown because they are contingent on thinking, deciding and acting of all interconnected economic agents. This is an issue which was neglected in the unawareness papers presented here. However, there are interesting attempts to account for the social construction of the (subjectively perceived) state space. Here we refer to the work on interactive unawareness by Heifetz, Meier and Schipper (2006, 2008) [39, 40], and on epistemic game theory; cf. Brandenburger (2008) [8].

Finally, it may be noted that only a small number of authors proposing theoretical models of agents' choice behaviour under conditions of uncertainty are committed to making testable predictions that may be refuted in principle. This state of affairs conflicts with logical positivism's view that the falsification of hypotheses by means of observation and/or experiment is the primary method for attaining meaningful progress in any empirical scientific disciplines; see, e.g., Popper (2002) [55]. Ideally, future research in economics and decision theory will address this problem more carefully.

\section{References}

[1] Akerlof G A 1970 The market for "Lemons": quality uncertainty and the market mechanism The Quarterly Journal of Economics 84 488-500

[2] Akerlof G A and Shiller R J 2009 Animal Spirits: How Human Psychology Drives the Economy, and Why It Matters for Global Capitalism (Princeton, NJ: Princeton University Press)

[3] Beckert J 1996 What is sociological about economic sociology? Uncertainty and the embeddedness of economic action Theory and Society 25 803-840

[4] Bernoulli D 1738 Specimen theoriae novae de mensura sortis

English translation: 1954 Exposition of a new theory on the measurement of risk Econometrica 22 23-36

[5] Bewley T F 1986 Knightian decision theory: part I (Cowles Foundation: discussion paper)

[6] Bewley T F 2002 Knightian decision theory: part I Decisions in Economics and Finance 25 79-110

[7] Blume L, Easley D and Halpern J Y 2009 Constructive decision theory (Institute for Advanced Studies, Vienna: Economics Series) URL (cited on September 5, 2012): www.ihs.ac.at/publications/eco/es-246.pdf

[8] Brandenburger A 2008 Epistemic game theory: an overview The New Palgrave Dictionary of Economics Online eds S N Durlauf and L E Blume URL (cited on September 5, 2012): www.dictionaryofeconomics.com/article?id=pde2008E000257

[9] Callon M 1998 The Laws of the Market (Oxford: Blackwell)

[10] Colander D, Föllmer H, Haas A, Goldberg M D, Juselius K, Kirman A, Lux T and Sloth B 2009 The financial crisis and the systemic failure of academic economics (University of Copenhagen, Dept of Economics: discussion paper No. 09-03, March 9, 2009) URL (cited on August 28, 2012): http: //ssrn.com/abstract $=1355882$

[11] Crocco M 2002 The concept of degrees of uncertainty in Keynes, Shackle, and Davidson Nova Economia 12 11-28 
[12] Danielsson J and Shin H S 2003 Endogenous risk Modern Risk Management: A History ed P Field (London: Risk Books) 297-314

[13] Danielsson J, Shin H S and Zigrand J-P 2009 Risk appetite and endogenous risk (Financial Markets Group (LSE): FMG Discussion Papers DP647) URL (cited on September 5, 2012): www.princeton.edu/ hsshin/www/riskappetite.pdf

[14] Davidson P 1991 Is probability theory relevant for uncertainty? A post Keynesian perspective Journal of Economic Perspectives 5 129-143

[15] Dekel E, Lipman B L and Rustichini A 1998 Standard state-space models preclude unawareness Econometrica 66 159-173

[16] Dekel E, Lipman B L and Rustichini A 2001 Representing preferences with a unique subjective state space Econometrica 69 891-934

[17] Dequech D 2006 The new institutional economics and the theory of behaviour under uncertainty Journal of Economic Behavior and Organization 59 109-131

[18] Dewey J 1915 The logic of judgments of practise The Journal of Philosophy, Psychology and Scientific Methods 12505

[19] Diebold F X, Doherty N A and Herring R J 2010 The Known, the Unknown, and the Unknowable in Financial Risk Management: Measurement and Theory Advancing Practice (Princeton, NJ: Princeton University Press)

[20] The Economist 2007 A new fashion in modeling: what to do when you don't know everything URL (cited on August 28, 2012): www.economist.com/node/10172461

[21] Ellsberg D 1961 Risk, ambiguity, and the Savage axioms The Quarterly Journal of Economics 75 643-669

[22] Epstein L G and Wang T 1994 Intertemporal asset pricing under Knightian uncertainty Econometrica 62 283-322

[23] Epstein L G, Marinacci M and Seo K 2007 Coarse contingencies and ambiguity Theoretical Economics 2 355-394

[24] Epstein L G and Schneider M 2010 Ambiguity and asset markets Annual Reviews of Financial Economics 2 315-346

[25] Esposito E 2007 Die Fiktion der wahrscheinlichen Realität (Frankfurt am Main: Suhrkamp)

[26] Esposito E 2010 Die Zukunft der Futures: Die Zeit des Geldes in Finanzwelt und Gesellschaft (Heidelberg: Carl-Auer-Systeme)

[27] de Finetti B 1937 La prévision: ses lois logiques, ses sources subjectives Annales de l'Institut Henri Poincaré 7 1-68

[28] von Förster H 1993 KybernEthik (Berlin: Merve)

[29] Gilboa I 2009 Theory of Decision under Uncertainty (Cambridge: Cambridge University Press)

[30] Gilboa I and Schmeidler D 1989 Maxmin expected utility with non-unique prior Journal of Mathematical Economics 18 141-153

[31] Gilboa I, Postlewaite A W and Schmeidler D 2008 Probability and uncertainty in economic modeling Journal of Economic Perspectives 22 173-188 
[32] Grant S and Quiggin J 2007 Awareness and discovery (Houston, Rice University: working paper)

[33] Hansen L P, Sargent T J and Tallarini T D 1999 Robust permanent income and pricing Review of Economic Studies 66 873-907

[34] Hansen L P and Sargent T J 2001 Acknowledging misspecification in macroeconomic theory Review of Economic Dynamics 4 519-535

[35] Hansen L P and Sargent T J 2003 Robust control of forward-looking models Journal of Monetary Economics 50 581-604

[36] Hawking S W and Ellis G F R 1973 The Large Scale Structure of Space-Time (Cambridge: Cambridge University Press)

[37] Hayek F A 1945 The use of knowledge in society The American Economic Review 35 519-530

[38] Hayashi T 2012 Expanding state space and extension of beliefs Theory and Decision 73 591-604

[39] Heifetz A, Meier M and Schipper B C 2006 Interactive unawareness Journal of Economic Theory 130 78-94

[40] Heifetz A, Meier M and Schipper B C 2008 A canonical model for interactive unawareness Games and Economic Behavior 62 304-324

[41] IPCC Fourth Assessment Report: Climate Change 2007 URL (cited on September 2, 2012): www.ipcc.ch/publications_and_data/ar4/wg3/en/ch2s2-3.html

[42] Keynes J M 1921 A Treatise on Probability (London: Macmillan)

[43] Keynes J M 1937 The general theory of employment The Quarterly Journal of Economics 51 209-233

[44] Keynes J M, Bonar J and Lloyd I H 1926 Francis Ysidro Edgeworth 1845-1926 The Economic Journal 36 140-158

[45] Knight F H 1921 Risk, Uncertainty and Profit (Boston: Houghton Mifflin)

[46] Kreps D M 1979 A representation theorem for "preference for flexibility" Econometrica 47 565-577

[47] Kreps D M 1992 Static choice and unforeseen contingencies Economic Analysis of Markets and Games: Essays in Honor of Frank Hahn ed P Dasgupta, D Gale, O Hart and E Maskin (Cambridge, MA: MIT Press) 259-281

[48] Machina M J 2003 States of the world and the state of decision theory The Economics of Risk ed D Meyer (Kalamazoo, MI: W E Upjohn Institute for Employment Research) 17-49

[49] McGoey L 2012 Strategic unknowns: towards a sociology of ignorance Economy and Society 41 1-16

[50] Mackenzie D 2006 An Engine, Not a Camera: How Financial Models Shape Markets (Cambridge, MA: MIT Press)

[51] Nash S J 2003 On pragmatic philosophy and Knightian uncertainty Review of Social Economy $61251-272$

[52] Nehring K 1999 Preference for flexibility in a Savage framework Econometrica 67 101-119

[53] von Neumann J and Morgenstern O 1944 Theory of Games and Economic Behavior (Princeton, NJ: Princeton University Press) 
[54] Nishimura K G and Ozaki H 2004 Search and Knightian uncertainty Journal of Economic Theory 119 299-333

[55] Popper K R 2002 Conjectures and Refutations: The Growth of Scientific Knowledge $2^{\text {nd }}$ Edition (London: Routledge)

[56] Proctor R N 2008 Agnotology: the missing term to describe the cultural production of ignorance (and its study) Agnotology: The Making and Unmaking of Ignorance ed R N Proctor and L Schiebinger (Stanford, CA: Stanford University Press) 1-29

[57] Ramsey F P 1931 Truth and probability The Foundations of Mathematics and Other Logical Essays (London: Routledge and Kegan Paul) 156-198

[58] Rooney D, Hearn G and Ninan A (eds) 2008 Handbook on the Knowledge Economy (Cheltenham: Edward Elgar Publishing)

[59] Rigotti L and Shannon C 2005 Uncertainty and risk in financial markets Econometrica 73 203-243

[60] Sagi J S 2006 What is an 'endogenous state space'? Economic Theory 27 305-320

[61] Savage L J 1954 The Foundations of Statistics (New York: Wiley)

[62] Shackle G L S 1949 Expectations in Economics (Cambridge: Cambridge University Press)

[63] Shackle G L S 1955 Uncertainty in Economics and Other Reflections (Cambridge: Cambridge University Press)

[64] Shackle G L S 1959 Time and thought The British Journal for the Philosophy of Science 9 285-298

[65] Soros G 1998 The Crisis Of Global Capitalism: Open Society Endangered (New York: Public Affairs)

[66] Spence M A 1973 Job market signaling The Quarterly Journal of Economics 87 355-374

[67] Stigler G J 1961 The economics of information Journal of Political Economy 69 213-225

[68] Stiglitz J E 1975 The theory of 'screening', education, and the distribution of income The American Economic Review 65 283-300

[69] Stiglitz J E 2002 Information and the change in the paradigm in economics The American Economic Review 92 460-501

[70] Svetlova E and Fiedler M 2011 Understanding crisis: on the meaning of uncertainty and probability The Recession of 2008 - Competing Explanations eds O D Asenjo and C Marcuzzo (Camberley: Edward Elgar Publishing) 42-62

[71] Taleb N N 2007 The Black Swan - The Impact of the Highly Improbable (London: Penguin)

[72] Taleb N N 2010 Why did the crisis of 2008 happen? (SSRN: discussion paper) URL (cited on August 28, 2012): http://papers.ssrn.com/sol3/papers.cfm?abstract_id=1666042 